clinicians in line with Department of Health OIPP (Quality, Innovation, Productivity and Prevention) programme.

Aims To improve the quality of referrals and reduce inappropriate referrals to admissions unit and outpatients by educating GPs and nurses to be more competent and confident in management of children.

Methods 10 Masterclasses were run by 8 paediatric consultants/ PIP over a 6 week period in 2011. 125 GPs, 92 nurses (17 nurse practitioners, 28 Practice Nurses, 9 community nurses, 24 health visitors) and 29 others (triage, student nurses) attended these sessions. 8 topics including convulsions, gastro enteritis, respiratory infections and rashes covered the top ten non-elective admissions and outpatient referrals to hospital identified in survey.

Results Feedback showed that participants preferred small informal groups with an interactive format helpful for learning. $80 \%$ of 242 participants who completed evaluation forms found the Master-classes useful or extremely useful.

Conclusions Referral data for acute hospital admissions and outpatients for individual GPs before and after Masterclasses is currently being audited. A reduction of $5-10 \%$ in acute and outpatient referrals by GPs is expected by 2012.We aim to conduct paediatric masterclasses to include all primary care clinicians in the region.

\section{PREVALENCE OF FEBRILE SEIZURES IN CHILDREN ADMITTED TO TEACHING PUBLIC HOSPITAL}

doi:10.1136/archdischild-2012-302724.1690

'A Veiga, ${ }^{2} E 0$ Veiga, 'NV Moliterno, ${ }^{2} F M$ Moliterno, 'SS Cordeiro, 'SA Nogueira, ${ }^{3} \mathrm{~A}$ Siqueira, ${ }^{3}$ Cordebel. 'Paediatrics; ${ }^{2}$ Faculdade de Medicina de Petrópolis; ${ }^{3}$ Hospital Alcides Carneiro, Petrópolis, Brazil

Background and Aims Febrile seizure is the manifestation neuropediatric more frequent, occurring in $2 \%$ and $5 \%$ in childhood. It occurs in children between 6 months and 5 years, associated with fever in the absence of intracranial infection or other cause neurological defined and not preceded by afebrile seizures. It is usually generalized tonic-clonic, short, single, early and not accompanied by neurological phenomena after the crisis. The objective of this study was identify the prevalence of Febrile Convulsion in patients hospitalized for febrile seizures, in January 2006 to December 2010.

Methods Cross-sectional study of patients admitted to the pediatric ward in a teaching hospital in the State of Rio de Janeiro. We analyzed 174 patients admitted with seizures of any etiology.

Results Febrile seizures accounted for $36 \%$ of 174 cases of seizures and $1.11 \%$ of 5667 cases admitted in the five years of the study. The mean age was 2.2 years, ranging in age from 6 months and a maximum of six years. The mean hospital stay was 5.73 days. Males accounted for $68.25 \%$ of cases. Regarding the focus of fever, respiratory tract accounted for nearly $50 \%$ of cases being diagnosed with $23.76 \%$ and $19.04 \%$ of upper respiratory infections to pneumonia.

Conclusion This study confirms the prevalence of the epidemiological literature, predominantly male $(2: 1)$, average age between 6 months and 5 years and the respiratory tract as the main focus for febrile seizures. It is imperative that further studies to evaluate diagnosis, treatment and follow up cases.

\section{THE USE OF FATTY ACID SUPPLEMENTATION IN THE TREATMENT OF ADHD- IS THERE ANY EVIDENCE?}

doi:10.1136/archdischild-2012-302724.1691

1,2S Damodaran, ${ }^{3} \mathrm{G}$ Erumbala, ${ }^{4} \mathrm{D}$ Abraham, ${ }^{1} \mathrm{~L}$ Doddamani. 'Paediatrics, James Paget Hospital, Great Yarmouth; ${ }^{2}$ PICU, Addenbrookes Hospital, Cambridge; ${ }^{3}$ Paediatrics, Milton Keynes General Hospital NHS Foundation Trust, Milton Keynes; ${ }^{4}$ Neonatal Unit, Birmingham Women's Hospital, Birmingham, UK

Attention deficit hyperactivity disorder (ADHD) is the most common behavioural disorder in children. The mainstay of treatment is stimulant drugs. There is significant interest in the role of omega 3 fatty acids in ameliorating ADHD symptoms. We reviewed the evidence from available randomized controlled trials.

Clinical question In a child with ADHD (patient), will supplementation with LCPUFA (omega3) (intervention) improve symptoms (outcome)?

Sources Pubmed, Sumsearch, Ovid and Cochraine library. 7 randomised control trials were analysed.

Summary There was no uniformity in terms of the dose or duration of fatty acids. The criteria used for measuring outcome varied significantly. In the largest study, Sinn etal compared omega 3 with omega 3 and multivitamins and placebo. This study involved the longest treatment period. There was significant improvement in ability to switch and control attention. But, there was no improvement in any other cognitive measures. Richardson et al and Belanger et al reported statistically significant improvements in symptoms with higher doses of omega 3. However both used small sample size. The former study lacked robust inclusion criteria. Of the studies that used low dose regime, Gustafson et al demonstrated significant improvement in cognition, inattention and opposition while Voigt et al failed to show any. Interestingly, Hirayama et al showed improvement in the placebo group.

The current available evidence is not sufficient to support the use of omega 3 fatty acid in the treatment of ADHD. More studies need to be conducted in the future using objective outcome criteria and good sample size.

\section{THE AETIOLOGY OF ACUTE ABDOMINAL PAIN IN CHILDREN 2-12 YEARS OF AGE}

doi:10.1136/archdischild-2012-302724.1692

N Kandamany, MB 0'Neill. Mayo General Hospital, Castlebar, Ireland

Background and Aims Abdominal pain is common in childhood. This study evaluated abdominal pain in children aged 2-12 to determine the frequency of aetiologies.

Methods Patients between 2 and 12 years presenting to the Emergency Department over a 5 month period, with abdominal pain were included. Patients with chronic constipation, previous abdominal pain and previous gastrointestinal surgery were excluded. Data was collected prospectively utilising patient's charts and a pretested proforma on abdominal pain which contained diagnostic tips and prompts. Demographic data, referral source, symptom complex, examination findings, and diagnoses were noted. Patients with a diagnosis of constipation were followed up via telephone to confirm accuracy of diagnosis.

Results One hundred and thirteen patients were assessed .Aetiologies of abdominal pain were appendicitis 26 (23\%), constipation $23(20 \%)$, gastroenteritis $18(16.5 \%)$, mesenteric adenitis 15 (13\%),non specific abdominal pain(viral syndrome) 11 (10\%), urinary tract infection $7(6 \%)$, pneumonia $6(5 \%)$, gastritis 5 (4.4\%),acute asthma 2 (2\%). Eighty four (75\%) patients were referred by their family doctor. Patients diagnosed with constipation were contacted by telephone to confirm the diagnosis, with $18(78 \%)$ agreeing.

Conclusion This study outlines the aetiology of abdominal pain in a paediatric population, three quarters of whom had been assessed by their family doctors prior to referral. Non gastrointestinal aetiologies need to be considered in the assessment.

\section{3 \\ PRIAMRY CARE GIVERS OF INFANTS ON HOME OXYGEN}

doi:10.1136/archdischild-2012-302724.1693

${ }^{1} \mathrm{~T}$ Nzirawa, ${ }^{2 \mathrm{M}}$ Ahmed, ${ }^{2} \mathrm{~A}$ Haque. ${ }^{1}$ Neonatal Medicine; ${ }^{2}$ Paediatrics, Queen's University Hospital, London, UK 